\title{
Colorectal Cancer Protection and Colonoscopy
}

\author{
Shrestha UK ${ }^{*}$ \\ ${ }^{I}$ Department of Medicine, Manipal College of Medical Sciences, Pokhara, Nepal
}

\author{
* Corresponding Author: \\ Dr. Umid Kumar Shrestha, MD, PhD \\ Department of Medicine \\ Manipal College of Medical Sciences \\ \& Manipal Teaching Hospital \\ Pokhara, Nepal \\ Email: umidshrestha@gmail.com
}

\section{Citation}

Shrestha UK. Colorectal cancer protection and colonoscopy. Nepal Journal of Medical Sciences. 2012; 1(1): 1-2.
Colorectal cancer (CRC) is the third most commonly diagnosed cancer in males and the second in females, with over 1.2 million new CRC cases and 608,700 deaths estimated to have occurred in $2008 .^{1}$

Modifiable risk factors for CRC include smoking, physical inactivity, overweight and obesity, red and processed meat consumption, and excessive alcohol consumption. ${ }^{2}$ Moreover, age is the single most important risk factor for the disease and people who lead a healthy lifestyle can still develop polyps and cancer. The symptoms that might indicate colorectal cancer are blood in stools, narrower than normal stools, unexplained abdominal pain, change in bowel habits, anemia and weight loss, but there are often no symptoms until it is too late to treat; hence, CRC is also considered a silent killer. CRC can also be present in people with known family history, or predisposing conditions, such as inflammatory bowel disease.

CRC is one of the most preventable cancers because the majority arises from the precancerous polyp in the colon, which can be found during a screening procedure and removed before it turns into cancer. It is believed that screening has the potential to reduce CRC deaths by $60 \%{ }^{3}$ Screening of CRC is recommended between the age of 50 and 75 years with sigmoidoscopy every 5 years and colonoscopy every 10 years. ${ }^{4}$ People with risk factors, such as a family history of polyps, CRC or other risk factors, should begin screening at age 45. Apart from the endoscopic methods, other screening methods are stool blood tests such as fecal occult blood test (FOBT) or fecal immunochemical test (FIT), stool DNA, CT colonography (virtual colonoscopy), and barium enema. CT colonography appears as good as standard colonoscopy but is expensive and associated with radiation exposure. $^{5}$

Among the various methods of screening, colonoscopy is considered the preferred screening test because it is the only test that finds and removes precancerous polyps during the same procedure. With the other methods, if a polyp is found, that test must then be followed by a colonoscopy to remove the polyp. Colonoscopy with polypectomy can be associated with strongly reduced risk for $\mathrm{CRC}$ in the population setting. ${ }^{6}$ If a colonoscopy is normal in a person at average risk, no another 
screening colonoscopy would be needed for next 10 years. If a polyp or CRC is found, screening intervals may be more frequent.

CRC is occurring in high frequencies in many economically developing countries, in addition to the disproportionately high burden of cancers related to infections. A significant proportion of the worldwide burden of CRC could be prevented through the screening colonoscopy by means of early detection and treatment, as well as through the public health campaigns by promoting physical activity and healthier dietary patterns.

Keywords: Colorectal cancer screening; colonoscopy; polyp

\section{References:}

1. Jemal A, Bray F, Center MM, et al. Global cancer statistics. CA:A Cancer Journal for Clinicians 2011;61:69-90.

2. Ferrari $\mathrm{P}$, Jenab M, Norat $\mathrm{T}$, et al. Lifetime and baseline alcohol intake and risk of colon and rectal cancers in the European prospective investigation into cancer and nutrition (EPIC). Int $\mathrm{J}$ Cancer 2007;121:2065-72.

3. He J, Efron JE. Screening for colorectal cancer. Advances in surgery 2011;45:31-44.

4. Levin B, Lieberman DA, McFarland B, et al. Screening and Surveillance for the Early Detection of Colorectal Cancer and Adenomatous Polyps, 2008: A Joint Guideline From the American Cancer Society, the US Multi-Society Task Force on Colorectal Cancer, and the American College of Radiology. Gastroenterology 2008; 134:1570-95

5. Cunningham D, Atkin W, Lenz HJ, et al. Colorectal cancer. Lancet 2010; 375:1030-47.

6. Brenner H, Chang-Claude J, Seiler CM, et al. Protection from colorectal cancer after colonoscopy: a population-based, casecontrol study. Ann Intern Med 2011;154:22-30. 